\title{
On Diophantine quintuples
}

\author{
by \\ Andrej Dujella (Zagreb)
}

1. Introduction. The Greek mathematician Diophantus of Alexandria noted that the set $\left\{\frac{1}{16}, \frac{33}{16}, \frac{17}{4}, \frac{105}{16}\right\}$ has the following property: the product of any two of its distinct elements increased by 1 is a square of a rational number (see [5]). Fermat first found a set of four positive integers with the above property, and it was $\{1,3,8,120\}$.

Let $n$ be an integer. A set of positive integers $\left\{x_{1}, \ldots, x_{m}\right\}$ is said to have the property $D(n)$ if for all $1 \leq i<j \leq m$ the following holds: $x_{i} x_{j}+n=y_{i j}^{2}$, where $y_{i j}$ is an integer. Such a set is called a Diophantine $m$-tuple.

Davenport and Baker [4] showed that if $d$ is a positive integer such that the set $\{1,3,8, d\}$ has the property of Diophantus, then $d$ has to be 120 . This implies that the Diophantine quadruple $\{1,3,8,120\}$ cannot be extended to the Diophantine quintuple with the property $D(1)$. Analogous result was proved for the Diophantine quadruple $\{2,4,12,420\}$ with the property $D(1)$ [17], for the Diophantine quadruple $\{1,5,12,96\}$ with the property $D(4)$ [15] and for the Diophantine quadruples $\left\{k-1, k+1,4 k, 16 k^{3}-4 k\right\}$ with the property $D(1)$ for almost all positive integers $k$ [9].

Euler proved that every Diophantine pair $\left\{x_{1}, x_{2}\right\}$ with the property $D(1)$ can be extended in infinitely many ways to the Diophantine quadruple with the same property (see [12]). In [6] it was proved that the same conclusion is valid for the pair with the property $D\left(l^{2}\right)$ if the additional condition that $x_{1} x_{2}$ is not a perfect square is fulfilled.

Arkin, Hoggatt and Straus [3] proved that every Diophantine triple with the property $D(1)$ can be extended to the Diophantine quadruple. More precisely, if $x_{i} x_{j}+1=y_{i j}^{2}$, then we can set $x_{4}=x_{1}+x_{2}+x_{3}+2 x_{1} x_{2} x_{3}+$ $2 y_{12} y_{13} y_{23}$. For the Diophantine quadruple obtained in this way, they proved the existence of a positive rational number $x_{5}$ with the property that $x_{i} x_{5}+1$ is a square of a rational number for $i=1, \ldots, 4$.

1991 Mathematics Subject Classification: 11D09, 11D99. 
Using this construction, in $[2,7,8,11]$ some formulas for Diophantine quintuples in terms of polynomials, Fibonacci, Lucas, Pell and Pell-Lucas numbers were obtained.

In the present paper we prove that for all positive rational numbers $q, x_{1}, x_{2}, x_{3}, x_{4}$ such that $x_{i} x_{j}+q^{2}=y_{i j}^{2}, y_{i j} \in \mathbb{Q}$, for $1 \leq i<j \leq 4$, and $x_{1} x_{2} x_{3} x_{4} \neq q^{4}$, there exists a positive rational number $x_{5}$ such that $x_{i} x_{5}+q^{2}$ is a square of a rational number for $i=1, \ldots, 4$. As a corollary we get the result that for all Diophantine quadruples $\left\{x_{1}, x_{2}, x_{3}, x_{4}\right\}$ with the property $D(1)$ there exists a rational number $x_{5}$ such that $x_{i} x_{5}+1$ is a square of a rational number for $i=1, \ldots, 4$.

\section{Extension of Diophantine quadruples}

TheOREM 1. Let $q, x_{1}, x_{2}, x_{3}, x_{4}$ be rational numbers such that $x_{i} x_{j}+$ $q^{2}=y_{i j}^{2}, y_{i j} \in \mathbb{Q}$, for all $1 \leq i<j \leq 4$. Assume that $x_{1} x_{2} x_{3} x_{4} \neq q^{4}$. Then the rational number $x_{5}=A / B$, where

$$
\begin{gathered}
A=q^{3}\left[2 y_{12} y_{13} y_{14} y_{23} y_{24} y_{34}+q x_{1} x_{2} x_{3} x_{4}\left(x_{1}+x_{2}+x_{3}+x_{4}\right)\right. \\
\left.+2 q^{3}\left(x_{1} x_{2} x_{3}+x_{1} x_{2} x_{4}+x_{1} x_{3} x_{4}+x_{2} x_{3} x_{4}\right)+q^{5}\left(x_{1}+x_{2}+x_{3}+x_{4}\right)\right], \\
B=\left(x_{1} x_{2} x_{3} x_{4}-q^{4}\right)^{2},
\end{gathered}
$$

has the property that $x_{i} x_{5}+q^{2}$ is a square of a rational number for $i=$ $1, \ldots, 4$. To be more precise, for $i \in\{1,2,3,4\}$ we have $x_{i} x_{5}+q^{2}=\left(q \frac{x_{i} y_{j k} y_{j l} y_{k l}+q y_{i j} y_{i k} y_{i l}}{x_{1} x_{2} x_{3} x_{4}-q^{4}}\right)^{2}, \quad$ where $\{i, j, k, l\}=\{1,2,3,4\}$.

Proof. Let $i \in\{1,2,3,4\}$ and $\{i, j, k, l\}=\{1,2,3,4\}$. Then

$$
\begin{array}{rl}
\left(x_{1} x_{2} x_{3} x_{4}-q^{4}\right)^{2}\left(x_{i} x_{5}+q^{2}\right) \\
=2 & 2 q^{3} x_{i} y_{12} y_{13} y_{14} y_{23} y_{24} y_{34}+q^{4} x_{1} x_{2} x_{3} x_{4} x_{i}\left(x_{1}+x_{2}+x_{3}+x_{4}\right) \\
& +2 x_{i} q^{6}\left(x_{1} x_{2} x_{3}+x_{1} x_{2} x_{4}+x_{1} x_{3} x_{4}+x_{2} x_{3} x_{4}\right) \\
& +x_{i} q^{8}\left(x_{1}+x_{2}+x_{3}+x_{4}\right)+q^{2} x_{1}^{2} x_{2}^{2} x_{3}^{2} x_{4}^{2}-2 q^{6} x_{1} x_{2} x_{3} x_{4}+q^{10} \\
= & q^{2}\left[2 q x_{i} y_{12} y_{13} y_{14} y_{23} y_{24} y_{34}+q^{2} x_{i}^{2} x_{j} x_{k} x_{l}\left(x_{i}+x_{j}+x_{k}+x_{l}\right)\right. \\
& +2 q^{4} x_{i}^{2}\left(x_{j} x_{k}+x_{j} x_{l}+x_{k} x_{l}\right)+2 q^{4} x_{i} x_{j} x_{k} x_{l}+q^{6} x_{i}^{2} \\
& \left.+q^{6}\left(x_{i} x_{j}+x_{i} x_{k}+x_{i} x_{l}\right)+x_{i}^{2} x_{j}^{2} x_{k}^{2} x_{l}^{2}-2 q^{4} x_{i} x_{j} x_{k} x_{l}+q^{8}\right] \\
= & q^{2}\left[2 q x_{i} y_{12} y_{13} y_{14} y_{23} y_{24} y_{34}+x_{i}^{2}\left(x_{j} x_{k}+q^{2}\right)\left(x_{j} x_{l}+q^{2}\right)\left(x_{k} x_{l}+q^{2}\right)\right. \\
& \left.+q^{2}\left(x_{i} x_{j}+q^{2}\right)\left(x_{i} x_{k}+q^{2}\right)\left(x_{i} x_{l}+q^{2}\right)\right] \\
= & q^{2}\left(2 q x_{i} y_{12} y_{13} y_{14} y_{23} y_{24} y_{34}+x_{i}^{2} y_{j k}^{2} y_{j l}^{2} y_{k l}^{2}+q^{2} y_{i j}^{2} y_{i k}^{2} y_{i l}^{2}\right) \\
= & {\left[q\left(x_{i} y_{j k} y_{j l} y_{k l}+q y_{i j} y_{i k} y_{i l}\right)\right]^{2},}
\end{array}
$$

which proves the theorem. 
Since the signs of $y_{i j}$ are arbitrary, we have two choices for $x_{5}$. Let $x_{5}^{+}$and $x_{5}^{-}$denote these two numbers, and let $x_{5}^{+}$be the number which corresponds to the case where all $y_{i j}$ are nonnegative.

Corollary 1. Let $\left\{x_{1}, x_{2}, x_{3}, x_{4}\right\} \subset \mathbb{N}$ be the set with the property $D(1)$. Then there exists a rational number $x_{5}, 0<x_{5}<1$, such that $x_{i} x_{5}+1$ is a square of a rational number for $i=1, \ldots, 4$.

Proof. We claim that the number $x_{5}^{+}$, obtained by applying the construction from Theorem 1 to the set $\left\{x_{1}, x_{2}, x_{3}, x_{4}\right\}$ has the desired property. Indeed, it is sufficient to prove that $x_{5}^{+}<1$. Let us introduce the following notation:

$$
\begin{aligned}
\sigma_{1} & =x_{1}+x_{2}+x_{3}+x_{4}, \\
\sigma_{2} & =x_{1} x_{2}+x_{1} x_{3}+x_{1} x_{4}+x_{2} x_{3}+x_{2} x_{4}+x_{3} x_{4}, \\
\sigma_{3} & =x_{1} x_{2} x_{3}+x_{1} x_{2} x_{4}+x_{1} x_{3} x_{4}+x_{2} x_{3} x_{4}, \\
\sigma_{4} & =x_{1} x_{2} x_{3} x_{4}, \\
X & =\sigma_{1} \sigma_{4}+2 \sigma_{3}+\sigma_{1}, \\
Y & =y_{12} y_{13} y_{14} y_{23} y_{24} y_{34} .
\end{aligned}
$$

The proof that

is completed by showing that

$$
x_{5}^{+}=\frac{2 Y+X}{\left(\sigma_{4}-1\right)^{2}}<1
$$

$$
2 X<\left(\sigma_{4}-1\right)^{2} \text { and } 4 Y<\left(\sigma_{4}-1\right)^{2} .
$$

Without loss of generality we can assume that $x_{1}<x_{2}<x_{3}<x_{4}$. If $x_{1}=1$, then $x_{2} \neq 2$. Therefore, $x_{2} \geq 3, x_{3} \geq 4$ and $x_{4} \geq 5$. Hence $\sigma_{4} \geq 60$. Furthermore, from

$$
\frac{1}{x_{1} x_{2} x_{3}}+\frac{1}{x_{1} x_{2} x_{4}}+\frac{1}{x_{1} x_{3} x_{4}}+\frac{1}{x_{2} x_{3} x_{4}} \leq \frac{13}{60}<\frac{1}{4}
$$

it follows that $52 \leq 4 \sigma_{1}<\sigma_{4}$. In the same manner we can see that $59 \leq$ $\sigma_{2}<\sigma_{4}$ and $107 \leq \sigma_{3}<2 \sigma_{4}$ (see also [12]). Hence

$$
\left(\sigma_{4}-1\right)^{2}-2 X>\sigma_{4}^{2}-2 \sigma_{4}+1-\frac{\sigma_{4}^{2}}{2}-8 \sigma_{4}-\frac{\sigma_{4}}{2}=\frac{1}{2}\left(\sigma_{4}^{2}-21 \sigma_{4}+2\right)>0
$$

(since $\sigma_{4} \geq 60$ ). To get the second inequality from (1), we note that

$$
\begin{aligned}
Y^{2} & =\left(x_{1} x_{2}+1\right)\left(x_{1} x_{3}+1\right)\left(x_{1} x_{4}+1\right)\left(x_{2} x_{3}+1\right)\left(x_{2} x_{4}+1\right)\left(x_{3} x_{4}+1\right) \\
& =\sigma_{4}^{3}+\sigma_{2} \sigma_{4}^{2}-\sigma_{4}^{2}+\sigma_{1} \sigma_{3} \sigma_{4}+\sigma_{1}^{2} \sigma_{4}-2 \sigma_{2} \sigma_{4}+\sigma_{3}^{2}-\sigma_{4}+\sigma_{1} \sigma_{3}+\sigma_{2}+1 \\
& <\sigma_{4}^{3}+\sigma_{4}^{3}-\sigma_{4}^{2}+\frac{\sigma_{4}^{3}}{2}+\frac{\sigma_{4}^{3}}{16}-118 \sigma_{4}+4 \sigma_{4}^{2}-\sigma_{4}+\frac{\sigma_{4}^{2}}{2}+\sigma_{4}+1 \\
& =\frac{41}{16} \sigma_{4}^{3}+\frac{7}{2} \sigma_{4}^{2}-118 \sigma_{4}+1 .
\end{aligned}
$$


Therefore,

$$
\begin{aligned}
\left(\sigma_{4}-1\right)^{4}-16 Y^{2} & >\sigma_{4}^{4}-4 \sigma_{4}^{3}+6 \sigma_{4}^{2}-4 \sigma_{4}+1-41 \sigma_{4}^{3}-56 \sigma_{4}^{2}+1888 \sigma_{4}-16 \\
& =\sigma_{4}^{4}-45 \sigma_{4}^{3}-50 \sigma_{4}^{2}+1884 \sigma_{4}-15>0
\end{aligned}
$$

(since $\sigma_{4} \geq 60$ ), which completes the proof.

Corollary 2. Let $q, x_{1}, x_{2}, x_{3}$ be rational numbers such that $x_{i} x_{j}+q^{2}=$ $y_{i j}^{2}, y_{i j} \in \mathbb{Q}$ for all $1 \leq i<j \leq 3$. Let

$$
\begin{aligned}
& x_{4}=\left[2 y_{12} y_{13} y_{23}+2 x_{1} x_{2} x_{3}+q^{2}\left(x_{1}+x_{2}+x_{3}\right)\right] / q^{2}, \\
& x_{5}=\frac{4 y_{12} y_{13} y_{23}\left(x_{1} y_{23}+y_{12} y_{13}\right)\left(x_{2} y_{13}+y_{12} y_{23}\right)\left(x_{3} y_{12}+y_{13} y_{23}\right)}{\left(x_{1} x_{2} x_{3} x_{4}-q^{4}\right)^{2}} .
\end{aligned}
$$

Then the set $\left\{x_{1}, x_{2}, x_{3}, x_{4}, x_{5}\right\}$ has the property that the product of any two distinct elements of it increased by $q^{2}$ is equal to the square of a rational number. In the notation of Theorem 1, we have

$$
x_{5}=\frac{4 q^{3} y_{12} y_{13} y_{14} y_{23} y_{24} y_{34}}{\left(x_{1} x_{2} x_{3} x_{4}-q^{4}\right)^{2}} .
$$

Proof. Let $z_{1}=x_{1}, z_{2}=x_{2}, z_{3}=x_{3}, z_{4}=0$. Then the rational numbers $z_{1}, z_{2}, z_{3}, z_{4}$ satisfy the conditions of Theorem 1 , and its application gives us the number

$$
z_{5}=\left[2 y_{12} y_{13} y_{23}+2 x_{1} x_{2} x_{3}+q^{2}\left(x_{1}+x_{2}+x_{3}\right)\right] / q^{2} .
$$

Set $x_{4}=z_{5}$. We can now apply Theorem 1 to the numbers $x_{1}, x_{2}, x_{3}, x_{4}$. Let $x_{5}$ be the number which is obtained by this construction. Observe that, by Theorem 1 , for all $i \in\{1,2,3\}$,

$$
q y_{i 4}=x_{i} y_{j k}+y_{i j} y_{i k},
$$

where $\{i, j, k\}=\{1,2,3\}$. Let us introduce the following notation:

$$
\begin{gathered}
\Sigma_{1}=x_{1}+x_{2}+x_{3}, \quad \Sigma_{2}=x_{1} x_{2}+x_{1} x_{3}+x_{2} x_{3}, \quad \Sigma_{3}=x_{1} x_{2} x_{3}, \\
V=y_{12} y_{13} y_{23}, \quad W=y_{14} y_{24} y_{34} .
\end{gathered}
$$

We have

$$
V^{2}=\left(x_{1} x_{2}+q^{2}\right)\left(x_{1} x_{3}+q^{2}\right)\left(x_{2} x_{3}+q^{2}\right)=\Sigma_{3}^{2}+q^{2} \Sigma_{1} \Sigma_{3}+q^{4} \Sigma_{2}+q^{6} .
$$

From (2) it follows that

$$
\begin{aligned}
q^{3} W & =\left(x_{1} y_{23}+y_{12} y_{13}\right)\left(x_{2} y_{13}+y_{12} y_{23}\right)\left(x_{3} y_{12}+y_{13} y_{23}\right) \\
& =4 \Sigma_{3}^{2}+3 q^{2} \Sigma_{1} \Sigma_{3}+2 q^{4} \Sigma_{2}+q^{6}+V\left(4 \Sigma_{3}+q^{2} \Sigma_{1}\right) .
\end{aligned}
$$

Now it is easy to check that, in the notation of Corollary 1 ,

$$
q^{4} \sigma_{1} \sigma_{4}+2 q^{6} \sigma_{3}+q^{8} \sigma_{1}=2 q^{3} V W .
$$


Consequently,

$$
\begin{aligned}
x_{5} & =\frac{4 q^{3} V W}{\left(x_{1} x_{2} x_{3} x_{4}-q^{4}\right)^{2}}=\frac{4 q^{3} y_{12} y_{13} y_{14} y_{23} y_{24} y_{34}}{\left(x_{1} x_{2} x_{3} x_{4}-q^{4}\right)^{2}} \\
& =\frac{4 y_{12} y_{13} y_{23}\left(x_{1} y_{23}+y_{12} y_{13}\right)\left(x_{2} y_{13}+y_{12} y_{23}\right)\left(x_{3} y_{12}+y_{13} y_{23}\right)}{\left(x_{1} x_{2} x_{3} x_{4}-q^{4}\right)^{2}} .
\end{aligned}
$$

Let us now consider the question when one or both (since $x_{5}^{+}$and $x_{5}^{-}$ can be equal) of the numbers $x_{5}^{+}$and $x_{5}^{-}$will be equal to zero. For obvious reasons, such an extension of a Diophantine quadruple will be called trivial. We will see that the answer to this question is closely connected to the construction of Corollary 2. From now on, we assume that $q \neq 0$.

Proposition 1. In the notation of Theorem 1 , we have $x_{5}^{+}=x_{5}^{-}=0$ if and only if there exist $1 \leq i<j \leq 4$ such that $x_{i} x_{j}=-q^{2}$ and $x_{i}+x_{j}=$ $x_{k}+x_{l}$, where $\{i, j, k, l\}=\{1,2,3,4\}$.

Pr o of. From $x_{5}^{+}=x_{5}^{-}$we conclude that there exist $1 \leq i<j \leq 4$ such that $y_{i j}=0$, i.e. $x_{i} x_{j}=-q^{2}$. Substituting this into the expression for $x_{5}$ we obtain

$$
x_{5}=\frac{q^{2}\left(x_{i}+x_{j}-x_{k}-x_{l}\right)}{x_{k} x_{l}+q^{2}} .
$$

Consequently, the condition $x_{5}=0$ implies that $x_{i}+x_{j}=x_{k}+x_{l}$.

Conversely, suppose that $x_{1}, x_{2}, x_{3}, x_{4}$ satisfy the condition of the proposition. Then $y_{i j}=0$, and (4) implies that $x_{5}^{+}=x_{5}^{-}=0$.

Proposition 2. In the notation of Theorem 1 , we have $0 \in\left\{x_{5}^{+}, x_{5}^{-}\right\}$if and only if there exists $i \in\{1,2,3,4\}$ such that

$$
x_{i}=\left[2 y_{j k} y_{j l} y_{k l}+2 x_{j} x_{k} x_{l}+q^{2}\left(x_{j}+x_{k}+x_{l}\right)\right] / q^{2},
$$

where $\{i, j, k, l\}=\{1,2,3,4\}$.

Pr o of. We can assume that $y_{i j} \neq 0$ since otherwise the assertion of the proposition follows from Proposition 1. If $x_{5}=0$, then $x_{i} x_{5}+q^{2}=q^{2}$ for $i=1, \ldots, 4$. Hence, if $0 \in\left\{x_{5}^{+}, x_{5}^{-}\right\}$, then Theorem 1 implies that for an appropriate choice of the sign of $y_{i j}$ we have

$$
x_{i} y_{j k} y_{j l} y_{k l}+q y_{i j} y_{i k} y_{i l}= \pm\left(x_{1} x_{2} x_{3} x_{4}-q^{4}\right),
$$

where $\{i, j, k, l\}=\{1,2,3,4\}$. Hence, there is no loss of generality in assuming that

$$
x_{1} y_{23} y_{24} y_{34}+q y_{12} y_{13} y_{14}=x_{2} y_{13} y_{14} y_{34}+q y_{12} y_{23} y_{24} \text {. }
$$

This gives $\left(x_{1} y_{34}-q y_{12}\right) y_{23} y_{24}=\left(x_{2} y_{34}-q y_{12}\right) y_{13} y_{14}$. Set $x_{1} y_{34}-q y_{12}=$ 
$\alpha y_{13} y_{14}$. Then $x_{2} y_{34}-q y_{12}=\alpha y_{23} y_{24}$, and so

$$
\begin{aligned}
\alpha\left(x_{1} y_{23} y_{24} y_{34}+q y_{12} y_{13} y_{14}\right) & =x_{1} y_{34}\left(x_{2} y_{34}-q y_{12}\right)+q y_{12}\left(x_{1} y_{34}-q y_{12}\right) \\
& =x_{1} x_{2} y_{34}^{2}-q^{2} y_{12}^{2}=x_{1} x_{2} x_{3} x_{4}-q^{4} .
\end{aligned}
$$

We thus get $\alpha= \pm 1$ and $x_{1} y_{34}-q y_{12}= \pm y_{13} y_{14}$. Squaring this relation we obtain

$x_{1}^{2} x_{3} x_{4}+q^{2} x_{1}^{2}+q^{2} x_{1} x_{2}+q^{4}-2 q x_{1} y_{12} y_{34}=x_{1}^{2} x_{3} x_{4}+q^{2} x_{1} x_{3}+q^{2} x_{1} x_{4}+q^{4}$, and (if $x_{1} \neq 0$ ) $2 y_{12} y_{34}=q\left(x_{1}+x_{2}-x_{3}-x_{4}\right)$. Squaring again we obtain the quadratic equation in $x_{4}$ :

$$
\begin{aligned}
q^{2} x_{4}^{2}-2 x_{4} & {\left[q^{2}\left(x_{1}+x_{2}+x_{3}\right)+2 x_{1} x_{2} x_{3}\right] } \\
& +q^{2}\left(x_{1}^{2}+x_{2}^{2}+x_{3}^{2}-2 x_{1} x_{2}-2 x_{1} x_{3}-2 x_{2} x_{3}-4 q^{2}\right)=0,
\end{aligned}
$$

with the solutions

$$
x_{4}=\left[q^{2}\left(x_{1}+x_{2}+x_{3}\right)+2 x_{1} x_{2} x_{3} \pm 2 y_{12} y_{13} y_{23}\right] / q^{2} .
$$

We have been working under the assumption that $x_{1} \neq 0$. Now suppose that $x_{1}=0$. In the same manner, using Corollary 2 , it can be proved that $x_{1}=0$ implies

$$
x_{4}=x_{2}+x_{3} \pm y_{23},
$$

which is exactly the relation (5) for $x_{1}=0$.

This proves one implication of the proposition. The opposite implication is a direct consequence of (3).

\section{Examples}

EXAMPLE 1. Let us first show that the condition $x_{1} x_{2} x_{3} x_{4} \neq q^{4}$ from Theorem 1 is not superfluous. Indeed, the set $\{25600,50625,82944,518400\}$ has the property $D\left(86400^{2}\right)$ and

$$
25600 \cdot 50625 \cdot 82944 \cdot 518400=86400^{4} .
$$

As an illustration of the situation from Proposition 1 let us consider the set $\{-25,25,-24,24\}$ with the property $D(625)$ and the set $\{-1,64,48,15\}$ with the property $D(64)$. In both cases the construction from Theorem 1 gives $x_{5}^{+}=x_{5}^{-}=0$.

From $[10,(13)]$, for $a=2$ and $k=3$, we obtain the Diophantine quadruple $\{2,20,44,72\}$ with the property $D(81)$. It is easy to check that this quadruple does not satisfy the conditions of Proposition 2. Therefore the numbers $x_{5}^{+}$and $x_{5}^{-}$are different from 0. Indeed, $x_{5}^{+}=\frac{4860}{169}$ and $x_{5}^{-}=$ $-\frac{1156680}{1054729}$. Using $x_{5}^{+}$, we obtain the Diophantine quintuple $\{338,3380,4860$, $7436,12168\}$ with the property $D\left(39^{4}\right)$.

If we apply the construction from Theorem 1 to Diophantus' original set $\left\{\frac{1}{16}, \frac{33}{16}, \frac{17}{4}, \frac{105}{16}\right\}$, we obtain $x_{5}^{+}=\frac{1557225246720}{42521^{2}}$ and $x_{5}^{-}=-\frac{4387246080}{42521^{2}}$. 
The definition of a Diophantine $m$-tuple can be extended to subsets of $\mathbb{Q}$. Let $q$ be a rational number. We call a set $A=\left\{x_{1}, \ldots, x_{m}\right\} \subset \mathbb{Q} \backslash\{0\}$ a (rational) Diophantine $m$-tuple with the property $D(q)$ if the product of any two distinct elements of $A$ increased by $q$ is equal to the square of a rational number. The construction of the rational Diophantine quintuple with the property $D(1)$ which extends a given Diophantine triple was described in [3]. That construction is equivalent to the construction from Corollary 2. But Theorem 1 enables extension of Diophantine quadruples which are not of the form $\left\{x_{1}, x_{2}, x_{3}, x_{4}\right\}$ from Corollary 2 . One such quadruple is the set $\{2,20,44,72\}$ from Example 1. Let us now examine two ways for generation of such Diophantine quadruples.

EXAMPLE 2. Let $\left\{x_{1}, x_{2}, x_{3}, x_{4}\right\} \subset \mathbb{Q}$ be an arbitrary set with the property $D\left(q^{2}\right)$ and let $x_{5} \in \mathbb{Q}$ be the number which is obtained by applying Theorem 1 to this set. Then $\left\{x_{2}, x_{3}, x_{4}, x_{5}\right\}$ also has the property $D\left(q^{2}\right)$, and we can apply Theorem 1 again. In this way we obtain $x_{6} \in \mathbb{Q}$ such that $\left\{x_{2}, x_{3}, x_{4}, x_{5}, x_{6}\right\}$ has the property $D\left(q^{2}\right)$.

For example, if $x_{1}=k-1, x_{2}=k+1, x_{3}=4 k$ and $x_{4}=16 k^{3}-4 k$, then $\left\{x_{1}, x_{2}, x_{3}, x_{4}\right\}$ has the property $D(1)([6$, p. 22]) and we obtain

$$
x_{5}=\frac{4 k(2 k-1)(2 k+1)\left(4 k^{2}-2 k-1\right)\left(4 k^{2}+2 k-1\right)\left(8 k^{2}-1\right)}{\left(64 k^{6}-80 k^{4}+16 k^{2}-1\right)^{2}},
$$

and $x_{6}=P(k) / Q(k)$, where

$$
\begin{aligned}
P(k)= & \left(8 k^{3}-4 k^{2}+1\right)\left(8 k^{3}+4 k^{2}-4 k-1\right)\left(8 k^{3}-12 k^{2}+1\right) \\
& \times\left(8 k^{4}+4 k^{3}-8 k^{2}-k+1\right)\left(32 k^{4}-8 k^{3}+28 k^{2}+3\right) \\
& \times\left(32 k^{4}+8 k^{3}-12 k^{2}+1\right)\left(32 k^{4}+24 k^{3}-12 k^{2}-4 k+1\right) \\
& \times\left(32 k^{4}+40 k^{3}+4 k^{2}-4 k+1\right), \\
Q(k)= & \left(131072 k^{14}+131072 k^{13}-184320 k^{12}-180224 k^{11}+96256 k^{10}\right. \\
+ & 86016 k^{9}-26880 k^{8}-18432 k^{7}+4480 k^{6}+1792 k^{5}-480 k^{4} \\
- & \left.64 k^{3}+32 k^{2}-1\right)^{2} .
\end{aligned}
$$

It turns out that this factorization of the numerator of $x_{6}$ is not accidental. Namely, it can be checked that, in the notation of Theorem $1, x_{6}=P / Q$, where

$$
\begin{aligned}
P= & q^{3}\left(y_{12} y_{13} y_{14}+q y_{12} y_{13}+q y_{12} y_{23}+q y_{13} y_{23}\right) \\
& \times\left(y_{12} y_{13} y_{14}+q y_{12} y_{13}-q y_{12} y_{23}-q y_{13} y_{23}\right) \\
& \times\left(y_{12} y_{13} y_{14}-q y_{12} y_{13}+q y_{12} y_{23}-q y_{13} y_{23}\right) \\
& \times\left(y_{12} y_{13} y_{14}-q y_{12} y_{13}-q y_{12} y_{23}+q y_{13} y_{23}\right)\left(y_{23} y_{24}+y_{23} y_{34}+y_{24} y_{34}\right)
\end{aligned}
$$




$$
\begin{aligned}
& \times\left(y_{23} y_{24}+y_{23} y_{34}-y_{24} y_{34}\right)\left(y_{23} y_{24}-y_{23} y_{34}+y_{24} y_{34}\right) \\
& \times\left(-y_{23} y_{24}+y_{23} y_{34}+y_{24} y_{34}\right), \\
Q= & x_{1}^{4}\left(4 x_{2} x_{3} x_{4} y_{12} y_{13} y_{14} y_{23} y_{24} y_{34}-q x_{1}^{2} x_{2}^{2} x_{3}^{2} x_{4}^{2}+2 q^{5} x_{1} x_{2} x_{3} x_{4}-q^{9}\right)^{2} .
\end{aligned}
$$

Proposition 3. Let $x_{1}, x_{2}$ and $x_{3}$ be rational numbers such that the denominator of

$$
x_{4}=\frac{8\left(x_{3}-x_{1}-x_{2}\right)\left(x_{1}+x_{3}-x_{2}\right)\left(x_{2}+x_{3}-x_{1}\right)}{\left(x_{1}^{2}+x_{2}^{2}+x_{3}^{2}-2 x_{1} x_{2}-2 x_{1} x_{3}-2 x_{2} x_{3}\right)^{2}}
$$

is different from 0 . Then $x_{1} x_{4}+1, x_{2} x_{4}+1$ and $x_{3} x_{4}+1$ are squares of rational numbers.

Proof. It follows immediately that

$$
x_{1} x_{4}+1=\left(\frac{x_{2}^{2}-2 x_{2} x_{3}+x_{3}^{2}-3 x_{1}^{2}+2 x_{1} x_{2}+2 x_{1} x_{3}}{x_{1}^{2}+x_{2}^{2}+x_{3}^{2}-2 x_{1} x_{2}-2 x_{1} x_{3}-2 x_{2} x_{3}}\right)^{2},
$$

and analogous relations hold for $x_{2} x_{4}+1$ and $x_{3} x_{4}+1$.

EXAmple 3. Observe that the set $\left\{x_{1}, x_{2}, x_{3}\right\}$ in Proposition 3 does not need to have the property $D(1)$. Take for example $x_{1}=F_{2 n+1}, x_{2}=F_{2 n+3}$ and $x_{3}=F_{2 n+5}$. Then $\left\{x_{1}, x_{2}, x_{3}\right\}$ has the property $D(-1)$ for every positive integer $n$ (see $[13,14]$ ). Proposition 3 implies that there exists a rational number $x_{4}$ with the property that $x_{i} x_{4}+1, i=1,2,3$, are squares of rational numbers. We will show that in this case $x_{4}$ is an integer. Indeed,

$$
\begin{aligned}
x_{1}^{2}+x_{2}^{2}+x_{3}^{2} & -2 x_{1} x_{2}-2 x_{1} x_{3}-2 x_{2} x_{3} \\
& =\left(x_{1}-x_{2}+x_{3}\right)^{2}-4 x_{1} x_{3} \\
& =\left[F_{2 n+1}-F_{2 n+3}+\left(3 F_{2 n+3}-F_{2 n+1}\right)\right]^{2}-4 F_{2 n+1} F_{2 n+5} \\
& =4\left(F_{2 n+3}^{2}-F_{2 n+1} F_{2 n+5}\right)=-4 .
\end{aligned}
$$

Hence,

$$
x_{4}=\frac{8}{16} \cdot 2 F_{2 n+2} \cdot 2 F_{2 n+3} \cdot 2 F_{2 n+4}=4 F_{2 n+2} F_{2 n+3} F_{2 n+4} .
$$

EXAMPLE 4 . If $x_{1} x_{2}+1=y_{12}^{2}$ and $x_{3}=x_{1}+x_{2}+2 y_{12}$, then $\left\{x_{1}, x_{2}, x_{3}\right\}$ has the property $D(1)$. If we apply the construction from Proposition 3 to this set we obtain

$$
x_{4}=4 y_{12}\left(x_{1}+y_{12}\right)\left(x_{2}+y_{12}\right) .
$$

If we apply the construction from Corollary 2 we obtain exactly the same result.

EXAMPLE 5. Let $x_{1}=1, x_{2}=3$ and $x_{3}=120$. Then Proposition 3 gives $x_{4}=\frac{834968}{3361^{2}}$. The set $\left\{x_{1}, x_{2}, x_{3}, x_{4}\right\}$ has the property $D(1)$ and we can apply 
the construction from Theorem 1. We obtain

$$
x_{5}^{+}=\frac{3985166705520 \cdot 481^{2}}{601439^{2} \cdot 481^{2}}, \quad x_{5}^{-}=\frac{426360 \cdot 601439^{2}}{481^{2} \cdot 601439^{2}} .
$$

It turns out that this cancellation is not accidental. Namely, let $\left\{x_{1}, x_{2}, x_{3}\right\}$ be an arbitrary set with the property $D(1)$, let $x_{4}$ be obtained by applying Proposition 3 to this set, and let $x_{5}^{+}$and $x_{5}^{-}$be obtained by applying Theorem 1 to $\left\{x_{1}, x_{2}, x_{3}, x_{4}\right\}$. Then

$$
\sqrt{x_{1} x_{5}^{+}+1} \cdot \sqrt{x_{1} x_{5}^{-}+1}=\left|\frac{(a+b)(a-b) c d}{c^{2} d^{2}}\right|,
$$

where

$$
\begin{aligned}
& a=x_{1} y_{23}\left[x_{1}^{2}\left(4 x_{2} x_{3}+1\right)-2 x_{1}\left(x_{2}+x_{3}\right)\left(2 x_{2} x_{3}-1\right)-\left(3 x_{2}^{2}+2 x_{2} x_{3}+3 x_{2}^{2}\right)\right], \\
& b=y_{12} y_{13}\left[x_{1}^{2}\left(-4 x_{2} x_{3}-3\right)+2 x_{1}\left(x_{2}+x_{3}\right)\left(2 x_{2} x_{3}+1\right)+\left(x_{2}-x_{3}\right)^{2}\right], \\
& c=\left(x_{1}+x_{2}+x_{3}\right)^{2}-4\left(x_{1} x_{2} x_{3}-y_{12} y_{13} y_{23}\right)^{2}+4, \\
& d=4\left(x_{1} x_{2} x_{3}+y_{12} y_{13} y_{23}\right)^{2}-\left(x_{1}+x_{2}+x_{3}\right)^{2}-4 .
\end{aligned}
$$

For $x_{1}=1, x_{2}=3$ and $x_{3}=120$, we get $c=4 \cdot 481$ and $d=4 \cdot 601439$.

4. Some open problems. One question still unanswered is whether there exists a (positive integer) Diophantine quintuple with the property $D(1)$. Corollary 1 shows that if such a quintuple exists it cannot be obtained by the construction from Theorem 1 . Let us mention that the analogous result for the sets with the property $D\left(l^{2}\right)$, where $l>1$, does not hold. For example, if we apply the construction from Theorem 1 to the quadruples $\{4,21,69,125\}$ and $\{7,12,63,128\}$ with the property $D(400)$, we obtain $x_{5}^{+}=384, x_{5}^{-}=-\frac{4032000}{1129^{2}}$ and $x_{5}^{+}=375, x_{5}^{-}=-\frac{11856000}{2021^{2}}$, respectively. Hence, the sets $\{4,21,69,125,384\}$ and $\{7,12,63,128,375\}$ are Diophantine quintuples with the property $D(400)$.

One may ask what is the least positive integer $n_{1}$, and what is the greatest negative integer $n_{2}$, for which there exists a Diophantine quintuple with the property $D\left(n_{i}\right), i=1,2$. Certainly $n_{1} \leq 256$ and $n_{2} \geq-255$, since the sets $\{1,33,105,320,18240\}$ and $\{5,21,64,285,6720\}$ have the property $D(256)$, and the set $\{8,32,77,203,528\}$ has the property $D(-255)$.

In the present paper we have considered quintuples with the property $D(q)$, where $q$ was a square of a rational number. However, the last set with the property $D(-255)$ indicates that there exist quintuples with the property $D(q)$, where $q$ is not a perfect square (see also $[9,16]$ ). Thus we come to the following open problem: For what rational numbers $q$ does there exist a rational Diophantine quintuple with the property $D(q)$ ? It follows easily from [6, Theorem 5] that for every rational number $q$ there exists a rational Diophantine quadruple with the property $D(q)$. 
At present it is not known whether there exists a rational number $q \neq 0$ such that there exists a rational Diophantine sextuple with the property $D(q)$. In [1], some rational "sextuples" with the property $D(1)$ were obtained, but all of them have two equal elements. Thus, they are actually quintuples with the additional property that $x_{1}^{2}+1$ is a perfect square. There exists also a rational Diophantine quintuple $\left\{x_{1}, \ldots, x_{5}\right\}$ with the property $D(1)$ such that $x_{1}^{2}+1, x_{2}^{2}+1$ and $x_{3}^{2}+1$ are perfect squares. However, the question of the existence of Diophantine sextuples is still open.

\section{References}

[1] J. Arkin, D. C. Arney, F. R. Giordano, R. A. Kolb and G. E. Bergum, An extension of an old classical Diophantine problem, in: Application of Fibonacci Numbers, Vol. 5, G. E. Bergum, A. N. Philippou and A. F. Horadam (eds.), Kluwer, Dordrecht, 1993, 45-48.

[2] J. Arkin and G. E. Bergum, More on the problem of Diophantus, in: Application of Fibonacci Numbers, Vol. 2, A. N. Philippou, A. F. Horadam and G. E. Bergum (eds.), Kluwer, Dordrecht, 1988, 177-181.

[3] J. Arkin, V. E. Hoggatt and E. G. Straus, On Euler's solution of a problem of Diophantus, Fibonacci Quart. 17 (1979), 333-339.

[4] H. Davenport and A. Baker, The equations $3 x^{2}-2=y^{2}$ and $8 x^{2}-7=z^{2}$, Quart. J. Math. Oxford Ser. (2) 20 (1969), 129-137.

[5] Diophantus of Alexandria, Arithmetics and the Book of Polygonal Numbers, Nauka, Moscow, 1974 (in Russian).

[6] A. Dujella, Generalization of a problem of Diophantus, Acta Arith. 65 (1993), $15-27$.

[7] -, Diophantine quadruples for squares of Fibonacci and Lucas numbers, Portugal. Math. 52 (1995), 305-318.

[8] -, Generalized Fibonacci numbers and the problem of Diophantus, Fibonacci Quart. 34 (1996), 164-175.

[9] —, Generalization of the Problem of Diophantus and Davenport, Dissertation, University of Zagreb, 1996 (in Croatian).

[10] - Some polynomial formulas for Diophantine quadruples, Grazer Math. Ber. 328 (1996), 25-30.

[11] - A problem of Diophantus and Pell numbers, in: Application of Fibonacci Numbers, Vol. 7, Kluwer, Dordrecht, to appear.

[12] P. Heichelheim, The study of positive integers $(a, b)$ such that $a b+1$ is a square, Fibonacci Quart. 17 (1979), 269-274.

[13] V. E. Hoggatt and G. E. Bergum, A problem of Fermat and the Fibonacci sequence, ibid. 15 (1977), 323-330.

[14] C. Long and G. E. Bergum, On a problem of Diophantus, in: Application of Fibonacci Numbers, Vol. 2, A. N. Philippou, A. F. Horadam and G. E. Bergum (eds.), Kluwer, Dordrecht, 1988, 183-191.

[15] S. P. Mohanty and M. S. Ramasamy, The characteristic number of two simultaneous Pell's equations and its application, Simon Stevin 59 (1985), 203-214.

[16] V. K. Mootha, On the set of numbers $\{14,22,30,42,90\}$, Acta Arith. 71 (1995), 259-263. 
[17] M. Veluppillai, The equations $z^{2}-3 y^{2}=-2$ and $z^{2}-6 x^{2}=-5$, in: A Collection of Manuscripts Related to the Fibonacci Sequence, V. E. Hoggatt and M. BicknellJohnson (eds.), The Fibonacci Association, Santa Clara, 1980, 71-75.

Department of Mathematics

University of Zagreb

Bijenička cesta 30

10000 Zagreb, Croatia

E-mail: duje@math.hr 